\title{
Experimental Investigation of Coal Dust Wettability Based on Surface Contact Angle
}

\author{
Gang Zhou, ${ }^{1,2,3,4}$ Han Qiu, ${ }^{1,4}$ Qi Zhang, ${ }^{1,4}$ Mao Xu,, Jiayuan Wang, ${ }^{1,4}$ and Gang Wang \\ ${ }^{1}$ State Key Laboratory of Mining Disaster Prevention and Control Co-Founded by Shandong Province and the Ministry of Science \\ and Technology, Shandong University of Science and Technology, Qingdao 266590, China \\ ${ }^{2}$ College of Mining and Safety Engineering, Shandong University of Science and Technology, Qingdao 266590, China \\ ${ }^{3}$ Key Laboratory of Coal Mine Gas and Fire Prevention and Control of Ministry of Education, China University of Mining and \\ Technology, Xuzhou 221116, China \\ ${ }^{4}$ Key Laboratory of Safety and High-Efficiency Coal Mining of Ministry of Education, Anhui University of Science and Technology, \\ Huainan 232001, China
}

Correspondence should be addressed to Gang Zhou; zhougang@sdust.edu.cn

Received 30 March 2016; Revised 27 June 2016; Accepted 17 July 2016

Academic Editor: Thijs A. Peters

Copyright (C) 2016 Gang Zhou et al. This is an open access article distributed under the Creative Commons Attribution License, which permits unrestricted use, distribution, and reproduction in any medium, provided the original work is properly cited.

\begin{abstract}
Wettability is one of the key chemical properties of coal dust, which is very important to dedusting. In this paper, the theory of liquid wetting solid was presented firstly; then, taking the gas coal of Xinglongzhuang coal mine in China as an example, by determination of critical surface tension of coal piece, it can be concluded that only when the surface tension of surfactant solution is less than $45 \mathrm{mN} / \mathrm{m}$ can the coal sample be fully wetted. Due to the effect of particle dispersity, compared with the contact angle of milled coal particle, not all the contact angles of screened coal powder with different sizes have a tendency to increase. Furthermore, by the experiments of coal samples' specific surface areas and porosities, it can be achieved that the volume of single-point total pore decreases with the gradual decreasing of coal's porosity, while the ultramicropores' dispersities and multipoint BET specific surface areas increase. Besides, by a series of contact angle experiments with different surfactants, it can be found that with the increasing of porosity and the decreasing of volume percentage of ultramicropore, the contact angle tends to reduce gradually and the coal dust is much easier to get wetted.
\end{abstract}

\section{Introduction}

As an inevitable product during the productive process in coal mine, coal dust has four main hazards as follows: (1) workers who contact with dust for a long time are inclined to get pneumoconiosis. (2) Dust leads to dust explosion when meeting some certain conditions. (3) Dust can make the field visuality much worse, which will bring out other safety accidents. (4) Dust can accelerate the electromechanical equipment's wear and reduce the reliability of precision instrument. Among the above four hazards, the most serious ones are dust explosion and pneumoconiosis $[1,2]$. There were 14 dust explosion accidents that happened in coal mine of China, leading to 483 deaths from the beginning of 2000 to the end of 2015 [3]. From 1950s to now, the number of cumulative reported pneumoconiosis cases of coal mine in China is more than 743 thousand, among which there are more than 282 thousand deaths; more seriously, the reported pneumoconiosis number is as high as 11396 only in 2015 [4]. According to the incomplete statistics, the number of people who died of pneumoconiosis in Chinese coal mine is six times as those who died from other mine disasters or accidents; in addition, the medical expenses on treating pneumoconiosis can reach 8 billion RMB every year in China [5]. Therefore, research on dust prevention is of great importance for coal mine safety production and miners' physical and mental health.

Nowadays, spraying dedusting is still the most widely used dust-prevention measurement in coal mine, which not only is economic and convenient but also can get good 
dedusting effects, and in this way the average dust reduction rate can reach about $60 \%$, while there are many factors influencing the spraying dedusting effects, and the main ones include the physical and chemical properties of coal dust, spraying pressure, nozzle type, particle size of droplets, and sprayer arrangement, among where the coal dust wettability is one of the key factors. Therefore, conducting the research on the coal dust wettability is very significant to improve the spraying dedusting effects.

In coal mine dedusting field, studies about the coal dust wettability have been limited to measure the contact angle formed on the surface of suppressed coal powder test piece by distilled water for a long period; this method is somewhat simple and the research achievement is not much. The main research themes are shown as follows: (1) the relations among proximate analysis, element analysis of coal powder, and its wettability [6-9], (2) the influence of coal dust's surface structures on its wetting properties [10-13], and (3) coal dust wetting dynamic model [14-17]. However, the researches on the following aspects are rare: (1) comparative analysis of wettability between milled coal particle and coal powder with different sizes after screening, (2) study on the relations between porosity characters of coal and its wettability, which is outstanding in the application of coal seam water injection field, and (3) research on the relations between critical surface tension of coal dust and its wettability by contact angle method. Besides, generally the coal dust wettability is poor, so in spraying dedusting process surfactants are usually added to decrease the droplets' surface tensions to make the dust-prevention effects better $[18,19]$. Therefore, it is very necessary to analyze the wetting effect of different surfactants to coal dust.

Classical measuring methods of contact angle can be divided into three kinds: angle method, length method, and gravimetric method. Among them, angle method is easy to implement with high accuracy; thus it is very popular in coal dust wettability characterization [20]. Therefore, as for the weakness of the above research, in this paper, video optical contact angle measuring device has been used to conduct series of experiments on the contact angles formed by different surfactants and coal powder with different porosity characters; meanwhile, the critical surface tension of coal piece, the contact angles formed by different surfactants, and coal powder with different dispersion degrees before and after screening were tested, making the coal dust wetting mechanism improved and promoted.

\section{Theoretical Background}

2.1. The Concept of Liquid-Solid Interface and Wetting. The broad sense of wettability is the nature appearing during the process in which gas on solid surface is substituted by liquid. Liquid with different properties has different wettabilities for the same solid, while liquid with same property has different wettabilities for different solid.

Similar to the surface molecules of liquid, surface molecules of solid also have unsaturated intermolecular force. Because the solid surface is much more uneven than liquid

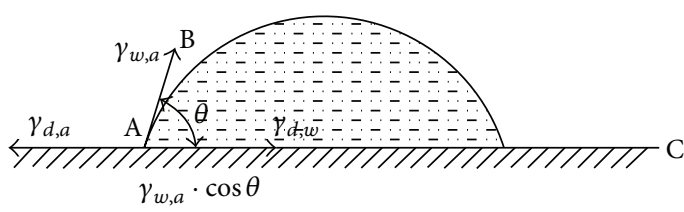

FIGURE 1: Schematic diagram about the wetting of dust particle expressed by surface tension.

surface, solid has much surface free enthalpy. Generally, liquid has the surface free enthalpy below $100 \mathrm{~mJ} / \mathrm{m}^{2}$, while solid surface can be divided into two kinds: surface with free enthalpy below $100 \mathrm{~mJ} / \mathrm{m}^{2}$ is called the low-energy surface, and those with free enthalpy above $100 \mathrm{~mJ} / \mathrm{m}^{2}$ or even above $500-5000 \mathrm{~mJ} / \mathrm{m}^{2}$ are called the high-energy surface. Therefore, the interface phenomenon will happen with the decrease in free enthalpy, as soon as the solid (especially the solid with high-energy surface) contacts with the surrounding mediums.

The solid interface exposed to the air is called the solid surface, which absorbs large amounts of gas. When solid surface contacts with liquid, the adsorption gas will be exhausted; meanwhile the liquid and solid interfaces are formed. In theory, this phenomenon is called wetting.

Assume that the surface tensions of liquid, solid, and the tension of liquid-solid interface are expressed by the forces along the surface and interface (as shown in Figure 1):

The process of wetting of dust particle can be expressed by the well-known Young equation:

$$
\gamma_{d, a}=\gamma_{d, w}+\gamma_{w, a} \cdot \cos \theta
$$

where $\theta$ is contact angle, ${ }^{\circ} ; \gamma_{d, a}$ is dust/gas interfacial tension, $\mathrm{mN} / \mathrm{m} ; \gamma_{d, w}$ is dust/water interfacial tension, $\mathrm{mN} / \mathrm{m} ; \gamma_{w, a}$ is water/gas interfacial tension, $\mathrm{mN} / \mathrm{m}$.

2.2. Wettability Criterion. Because of the wetting, new liquidsolid interface appears, taking place of the gas-solid interface, accompanied by changes of free enthalpy, which can be used for judging wetting. There are three kinds of wetting, as shown in Figure 2.

The criterions for different wetting types are as follows: spreading wetting: $\theta=0^{\circ}$; impregnation wetting: $\theta \leq 90^{\circ}$; adhesion wetting: $90^{\circ}<\theta \leq 180^{\circ}$. For the mineral dust, the wettability can be classified as the following three types by the value of contact angle [21]: water-wet: $\theta \leq 75^{\circ}$; intermediatewet: $75^{\circ}<\theta \leq 115^{\circ}$; gas-wet: $\theta>115^{\circ}$.

2.3. The Meaning of Critical Surface Tension. In reality, another criterion judging the wetting ability has been found, namely, $\Gamma_{c}$ which is the critical surface tension of solid. According to the experiments conducted by Zismann and some others, when determining the contact angle of liquid with different surface tensions on smooth and clean solid surface, the cosine of the contact angle has a linear relationship with the surface tension, which is called the Zismann curve. 


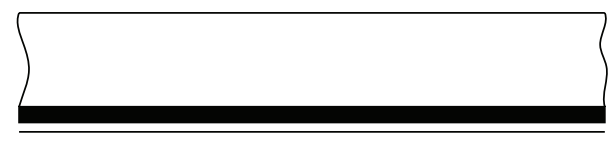

(a) Spreading wetting

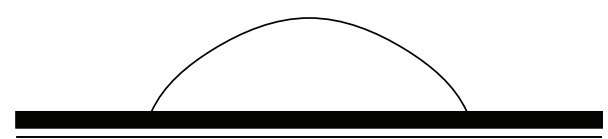

(b) Impregnation wetting

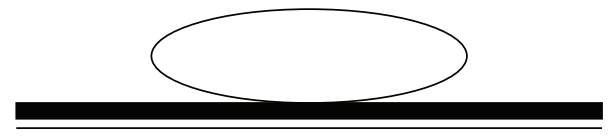

(c) Adhesion wetting

FIgURE 2: Wetting types.

Namely, when the liquid surface tension is greater than $\Gamma_{c}$ of a kind of solid, the liquid cannot fully wet the solid surface; otherwise, the solid surface could be fully wetted [22].

\section{Experimental Method}

3.1. Collection of the Coal Sample. The coal sample collected in Xinglongzhuang coal mine of Yankuang Group was gas coal. The results of proximate and elemental analysis of the coal sample were shown in Table 1.

3.2. Preparation of the Test Piece. Presently, there are two main ways to measure the contact angle on the coal pore surface. The first is to replace the pore surface with polished surface of coal; the second is to use the compressed surface of the shaped coal powder to take place of the pore surface. The brief introduction of the second method is as follows. To get the target coal powder, the coal sample was processed by a jaw crusher and a sealed pulverizer and then grinded with the ball mill for $30 \mathrm{~s}$. Then, the powder was firstly put into the vacuum drying oven to be dried for $2 \mathrm{~h}$ under $130^{\circ} \mathrm{C}$ to remove water. Secondly, $200 \mathrm{mg}$ powder was taken to remain under $30 \mathrm{MPa}$ condition for 2 minutes by using desktop powder tablet compressing machine and the matched ID13 cylindrical tablet mold. When removed out, the circular test piece with polished plane, which had a diameter of $13 \mathrm{~mm}$ and a thickness of $2 \mathrm{~mm}$, was achieved.

3.3. Contact Angle and Critical Surface Tension Measurements. The contact angle between surfactant solution and test piece was determined by DSA100 video optical contact angle measuring device. The device has functions such as automatically storing contact angle pictures (videos), automatically analyzing contact angle values, and automatically storing testing results. It not only has the advantages of clear picture, highly accurate testing result, and simple control, but also has additional functions such as measuring the surface tension of
TABLe 1: Proximate analysis and elemental analysis of the coal sample.

\begin{tabular}{ccccccccc}
\hline \multicolumn{3}{c}{ Proximate analysis/\% } & \multicolumn{5}{c}{ Elemental analysis/\% } \\
$\mathrm{M}_{\mathrm{ad}}$ & $\mathrm{A}_{\mathrm{ad}}$ & $\mathrm{V}_{\mathrm{ad}}$ & $\mathrm{FC}_{\mathrm{ad}}$ & $\mathrm{C}_{\mathrm{daf}}$ & $\mathrm{O}_{\mathrm{daf}}$ & $\mathrm{H}_{\mathrm{daf}}$ & $\mathrm{N}_{\mathrm{daf}}$ & $\mathrm{S}_{\mathrm{daf}}$ \\
\hline 5.26 & 7.35 & 34.72 & 52.67 & 81.61 & 11.16 & 5.77 & 1.15 & 0.31 \\
\hline
\end{tabular}

liquid solution by hanging drop method, as well as measuring the surface tension of solid using the surface energy method.

(1) Contact Angle Measurements. The status of distilled water droplets and wetting agents generated on the surface of coal samples has been imaged and analyzed by the optical contact angle meter (DSA100) via the methods of "contact angle using" and "circle fitting." The contour line of the wetting result is displayed on the image of the drops and all the measurement results of contact angle can be automatically acquired and displayed by the meter. And the measuring error of the instrument is $0.01^{\circ}$.

(2) Critical Surface Tension Measurements. When dropped on a surface of the coal sample, the liquid does not completely spread over but forms a certain angle with the solid. If liquids with different surface tensions are placed on the surface of the pressed specimen of pulverized coal and a DSA100 optical contact angle meter is used to determine the surface tension of droplets and the contact angles, different surface tensions have a linear relationship with the cosine of contact angles. When the line is nudged outwards to a contact angle with the cosine value of 1 , the corresponding surface tension is the critical surface tension between the solid and the liquid.

3.4. Determination of Coal Pore Characteristics. To study the relations among the contact angles formed on coal powder test pieces with different porosities, the pore characteristics of the samples from \#10301, \#1308, and \#7301 work face of Xinglongzhuang coal mine were firstly measured, using the SSA- 4000 series specific surface area and aperture analyzer based on the principle of low temperature liquidnitrogen adsorption in the static volumetric method. At low temperature, the adsorption of nitrogen follows physisorption. Mass balance equation and static gas pressure test were used to determine the porosity during the adsorption process $[23,24]$. The test is normally conducted at liquid-nitrogen temperature. A given volume of gas was filled in the sample tube via gas line. When the adsorption reached equilibrium, the molar mass of the adsorbed gas was calculated based on pressure drops and thus the adsorption-desorption isotherms were determined [25]. Then, the specific surface area and pore diameter distribution parameters of the samples can be equivalently obtained via relevant theoretical models $[26,27]$.

\section{Results and Discussion}

4.1. Determination of the Critical Surface Tension of Coal Sample. The critical surface tensions of the compressed test pieces made by coal powder from \#10301 work face of 


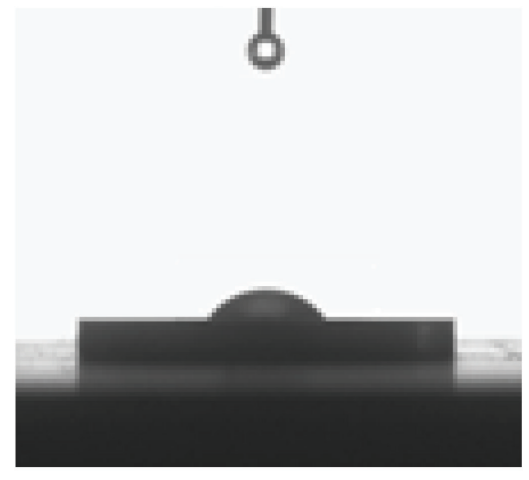

(a)

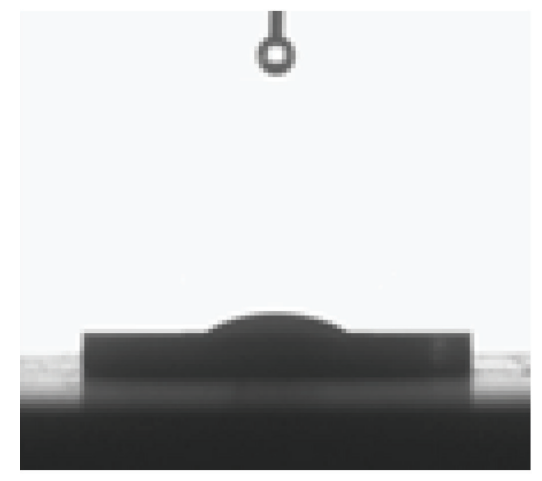

(b)

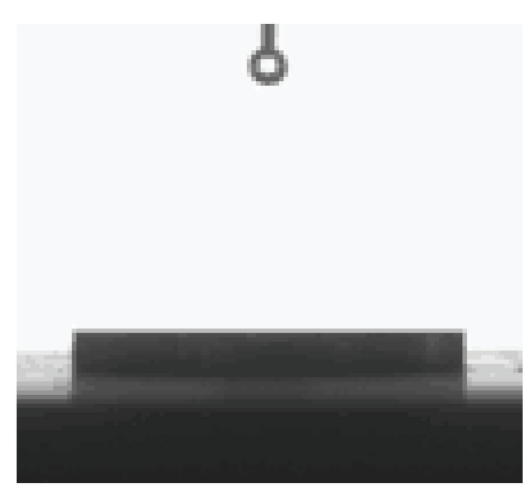

(c)

FIGURE 3: Effect of different SDS concentration on contact angle: (a) $0.005 \%$, (b) $0.05 \%$, and (c) $0.5 \%$ SDS.

Xinglongzhuang coal mine are measured by contact angle measuring device. According to the test results, the critical surface tension of the test piece made by dry coal powder was $44.85 \mathrm{mN} / \mathrm{m}$, but the one made by nondry coal powder was $49.77 \mathrm{mN} / \mathrm{m}$, which was greater than the former. Therefore, only when the surface tension of the surfactant solution was less than $45 \mathrm{mN} / \mathrm{m}$, according to the definition of $\Gamma_{c}$, could the coal be fully wetted; namely the contact angle would be $0^{\circ}$. On the other hand, for the same solution, the test piece made by nondry coal powder could be more easily wetted totally than that by dry coal powder, which was attributed to the changes of indexes such as moisture, volatiles, and ash before and after drying the coal.

4.2. Comparative Analysis of Contact Angles for Milled and Screened Coal Dust. The coal particle grinded by the ball mill for $30 \mathrm{~s}$ was, respectively, classified by the standard test sieve of 70 meshes (aperture of $0.224 \mathrm{~mm}$ ), 100 meshes (aperture of $0.154 \mathrm{~mm}), 160$ meshes (aperture of $0.094 \mathrm{~mm}), 200$ meshes (aperture of $0.074 \mathrm{~mm}$ ), and 320 meshes (aperture of $0.045 \mathrm{~mm}$ ). After being compressed, the test pieces of coal powder with different sizes were obtained. The coal sample was from 10301 work face of Xinglongzhuang coal mine, and the surfactants were, respectively, taken from one of the following four kinds: anionic, cationic, nonionic, or amphoteric. For example, the changing tendency of formed contact angles when the test pieces are wetted by the solution of sodium dodecyl sulfate (SDS) in different mass concentrations is shown in Figure 3. The total test data is shown in Table 2 and Figure 4.

According to Table 2 and Figure 4,

(1) compared with contact angles formed by coal particle milled for $30 \mathrm{~s}$, not all contact angles of coal powder screened by different meshes tended to increase. After being screened by 70 and 100 meshes, for coal pieces, their average changing values of contact angles decreased by $10.57^{\circ}$ and $4.27^{\circ}$, while the values increased by $2.01^{\circ}, 6.69^{\circ}$, and $10.12^{\circ}$, respectively, after being screened by 160, 200, and 320 meshes. The main reason was that the average granularity of coal particle

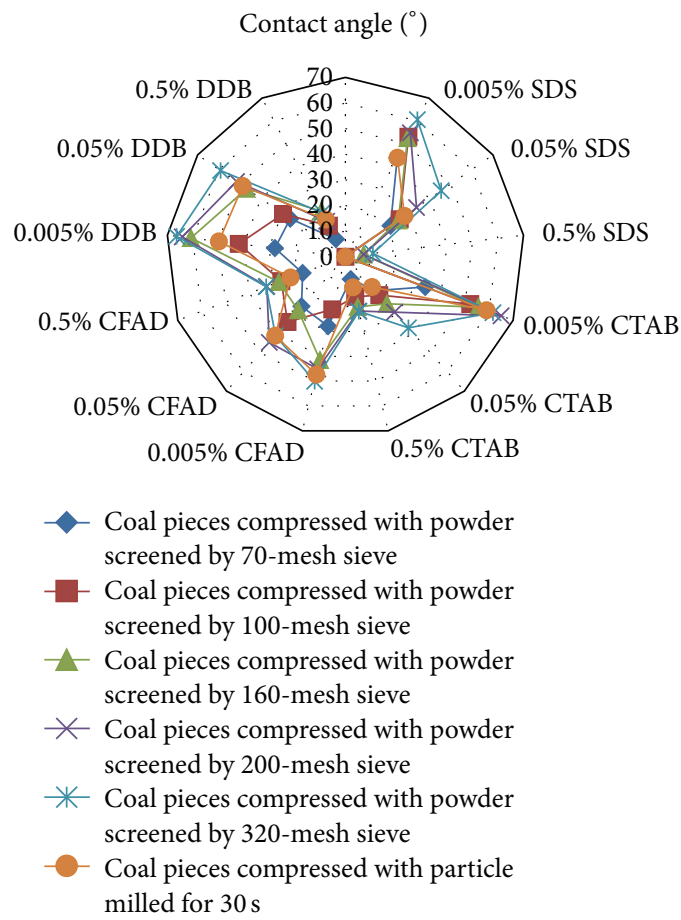

FIGURE 4: Comparison chart of contact angles among coal pieces with different powder sizes.

milled for $30 \mathrm{~s}$ was smaller than $0.15 \mathrm{~mm}$, and both large and small particle sizes have been distributed. However, after being screened by 70 and 100 meshes, the particle size was larger and the maximum size could reach $0.224 \mathrm{~mm}$, while the maximum size of coal powder screened by 160, 200, and 320 meshes could only reach $0.094 \mathrm{~mm}$;

(2) the wetting types of all the coal pieces with each particle size are impregnation wetting. With the increase in the number of sieving meshes, the contact angle tended to increase gradually. During the process that the particle size of coal powder decreased from $0.224 \mathrm{~mm}$ to $0.074 \mathrm{~mm}$, the average value of contact 
TABLE 2: Determination results of contact angles on test pieces of coal powder before and after being screened.

\begin{tabular}{|c|c|c|c|c|c|c|c|c|c|c|c|c|}
\hline \multirow{3}{*}{ Name of surfactants } & \multirow{3}{*}{ Concentration/\% } & \multicolumn{7}{|c|}{ Contact angle $/^{\circ}$} & \multirow{2}{*}{\multicolumn{4}{|c|}{$\begin{array}{c}\text { Change rates of the contact } \\
\text { angle } \\
\text { for different meshes/\% }\end{array}$}} \\
\hline & & \multicolumn{5}{|c|}{ Sieving meshes } & \multirow[t]{2}{*}{$\begin{array}{l}\text { Coal particle } \\
\text { milled for } 30 \mathrm{~s}\end{array}$} & & & & & \\
\hline & & 70 & 100 & 160 & 200 & 320 & & 70 & 100 & 160 & 200 & 320 \\
\hline \multirow{3}{*}{$\begin{array}{l}\text { Sodium dodecyl sulfate } \\
\text { (SDS)/anionic }\end{array}$} & 0.005 & 43.58 & 52.87 & 52.27 & 54.63 & 60.40 & 43.68 & -0.1 & 9.19 & 8.59 & 10.95 & 16.72 \\
\hline & 0.05 & 21.35 & 25.80 & 25.08 & 33.76 & 45.46 & 28.04 & -6.69 & -2.24 & -2.96 & 5.72 & 17.42 \\
\hline & 0.5 & 0 & 0 & 7.14 & 7.23 & 10.77 & 0 & 0 & 0 & 7.14 & 7.23 & 10.77 \\
\hline \multirow{3}{*}{$\begin{array}{l}\text { Cetyltrimethyl } \\
\text { ammonium bromide } \\
\text { (CTAB)/cationic }\end{array}$} & 0.005 & 33.36 & 52.16 & 55.47 & 64.94 & 61.63 & 58.97 & -25.61 & -6.81 & -3.5 & 5.97 & 2.66 \\
\hline & 0.05 & 17.71 & 19.94 & 24.44 & 28.95 & 37.09 & 15.74 & 1.97 & 4.2 & 8.7 & 13.21 & 21.35 \\
\hline & 0.5 & 9.03 & 16.08 & 20.16 & 21.79 & 21.96 & 12.28 & -3.25 & 3.8 & 7.88 & 9.51 & 9.68 \\
\hline \multirow{3}{*}{$\begin{array}{l}\text { Cocoanut fatty acid } \\
\text { diethanolamide } \\
(\mathrm{CFAD}) / \text { nonionic }\end{array}$} & 0.005 & 27.94 & 21.17 & 41.54 & 45.16 & 50.11 & 47.40 & -19.46 & -26.23 & -5.86 & -2.24 & 2.71 \\
\hline & 0.05 & 25.85 & 33.97 & 27.84 & 44.68 & 41.08 & 41.22 & -15.37 & -7.25 & -13.38 & 3.46 & -0.14 \\
\hline & 0.5 & 17.82 & 26.69 & 27.77 & 32.76 & 33.07 & 22.89 & -5.07 & 3.8 & 4.88 & 9.87 & 10.18 \\
\hline \multirow{3}{*}{$\begin{array}{l}\text { Dodecyl dimethyl betaine } \\
\text { (DDB)/amphoteric }\end{array}$} & 0.005 & 27.72 & 41.89 & 60.68 & 64.09 & 66.36 & 49.65 & -21.93 & -7.76 & 11.03 & 14.44 & 16.71 \\
\hline & 0.05 & 26.10 & 29.50 & 46.78 & 51.66 & 59.14 & 48.74 & -22.64 & -19.24 & -1.96 & 2.92 & 10.4 \\
\hline & 0.5 & 7.82 & 13.73 & 20.08 & 15.69 & 19.40 & 16.47 & -8.65 & -2.74 & 3.61 & -0.78 & 2.93 \\
\hline
\end{tabular}

angle formed by the four reagents increased from $21.52^{\circ}$ to $27.82^{\circ}, 34.10^{\circ}, 38.78^{\circ}$, and $42.21^{\circ}$ gradually;

(3) the anionic and cationic surfactants (SDS and CTAB) have better wetting ability than the nonionic and amphoteric surfactants (CFAD and DDB), which is similar with the contact angles formed on natural coal pieces, because the contact angles formed by the two former reagents were $28.45^{\circ}$ and $31.76^{\circ}$, respectively, which were smaller than $33.83^{\circ}$ and $36.97^{\circ}$ formed by the two latter reagents.

\subsection{Analysis of the Relationship between Pore Characteristics of Coal and Its Wettability}

4.3.1. Determination of Porosities of Different Coal Samples. Figure 5 shows the isotherm diagram of the nitrogen's absorption and desorption process about the sample from \#10301 work face. The summary of experimental data can be seen in Table 3.

According to the experiment [28], coal samples in different mining areas had different porosities, which gradually decreased from \#10301 work face to \#1308 work face and then to \#7301 work face. For example, the total pore volume of single-point, respectively, decreased from $0.0212 \mathrm{cc} / \mathrm{g}$ to $0.0136 \mathrm{cc} / \mathrm{g}$ and then to $0.0096 \mathrm{cc} / \mathrm{g}$. However, the multipoint BET specific surface area increased from $4.547 \mathrm{~m}^{2} / \mathrm{g}$ to $5.260 \mathrm{~m}^{2} / \mathrm{g}$ and then to $6.368 \mathrm{~m}^{2} / \mathrm{g}$. In addition, with the decrease in the total pore volume of single-point, the micropore volume of single-point adsorption also increased from $0.0024 \mathrm{cc} / \mathrm{g}$ to $0.0027 \mathrm{cc} / \mathrm{g}$ and then to $0.0031 \mathrm{cc} / \mathrm{g}$; namely, the dispersity of the ultramicropore decreased with the increase in the porosity.

4.3.2. Determination of Contact Angle of Coal Samples with Different Porosities. The selection of surfactant solution is

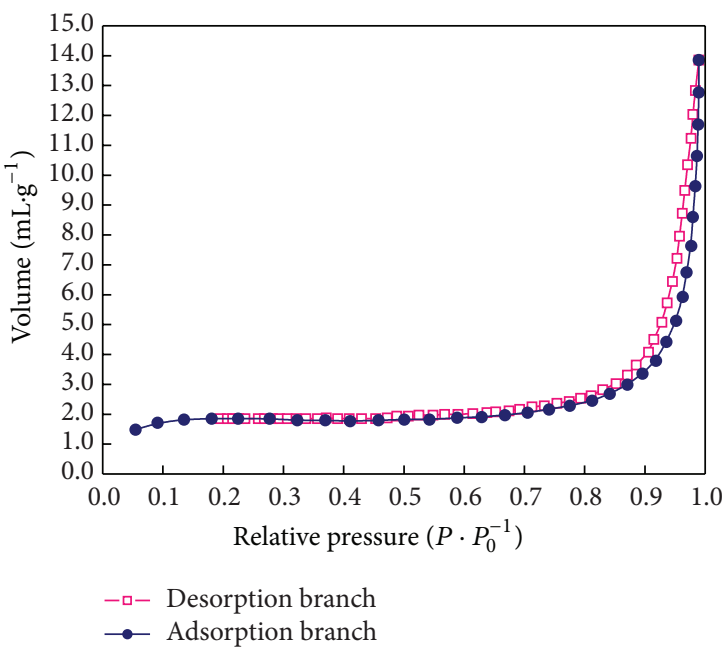

FIGURE 5: Isotherm diagram of the nitrogen's absorption and desorption process.

the same as the reagents in Table 2. Then, the contact angles on the polished coal samples were determined by the contact angle measuring device. Experimental data is shown in Table 4 and Figure 6, and the measuring error of the contact angle is $0.01^{\circ}$.

By analyzing data of porosities and contact angles, the relations between them were achieved. The relationships among contact angle and multipoint BET specific surface area, total pore volume of single-point, and micropore volume of single-point adsorption were shown in Figure 7.

According to Table 4 and Figures 6 and 7,

(1) for the same kind of reagent with same concentration, there is a big difference in the contact angle formed by coal samples with different porosities. But 
TABLE 3: Summary of experimental data about the porosity of coal sample in \#10301, \#1308, and \#7301 work face.

\begin{tabular}{|c|c|c|c|}
\hline Experiment items & $\# 10301$ & $\# 1308$ & $\# 7301$ \\
\hline Multipoint BET specific surface area $/\left(\mathrm{m}^{2} \cdot \mathrm{g}^{-1}\right)$ & 4.547 & 5.260 & 6.368 \\
\hline Specific surface area of BJH accumulative adsorption $/\left(\mathrm{m}^{2} \cdot \mathrm{g}^{-1}\right)$ & 0.861 & 0.790 & 1.882 \\
\hline Specific surface area of BJH accumulative desorption $/\left(\mathrm{m}^{2} \cdot \mathrm{g}^{-1}\right)$ & 1.841 & 1.362 & 2.753 \\
\hline Total pore volume of single-point/(cc. $\left.\mathrm{g}^{-1}\right)$ & 0.0212 & 0.0136 & 0.0096 \\
\hline Total pore volume of $\mathrm{BJH}$ accumulative adsorption $/\left(\mathrm{cc} \cdot \mathrm{g}^{-1}\right)$ & 0.0193 & 0.0092 & 0.0087 \\
\hline Total pore volume of $\mathrm{BJH}$ accumulative desorption $/\left(\mathrm{cc} \cdot \mathrm{g}^{-1}\right)$ & 0.0184 & 0.0087 & 0.0081 \\
\hline Micropore volume of single-point adsorption $/\left(\mathrm{cc} \cdot \mathrm{g}^{-1}\right)($ radius $<10 \AA)$ & 0.0024 & 0.0027 & 0.0031 \\
\hline Mean bore radius of single-point $/ \AA$ & 66.6 & 51.4 & 45.5 \\
\hline Mean bore radius of BJH adsorption/ $\AA$ & 206.0 & 221.5 & 210.1 \\
\hline Mean bore radius of $\mathrm{BJH}$ desorption/Å & 135.7 & 122.6 & 96.4 \\
\hline Most probable aperture of BJH adsorption/Å & 72.7 & 82.9 & 64.6 \\
\hline Most probable aperture of BJH desorption/Å & 19.4 & 19.0 & 18.3 \\
\hline
\end{tabular}

TABLE 4: Determination results of contact angles of coal samples with different porosities.

\begin{tabular}{|c|c|c|c|c|}
\hline \multirow{3}{*}{ Name of reagents } & \multicolumn{3}{|c|}{ Contact angle $^{\circ}$} & \\
\hline & \multirow{2}{*}{ Concentration/\% } & \multicolumn{3}{|c|}{ Coal samples from different work faces } \\
\hline & & $\# 10301$ & $\# 1308$ & \#7301 \\
\hline \multirow{3}{*}{ Sodium dodecyl sulfate (SDS)/anionic } & 0.005 & 16.73 & 19.43 & 26.35 \\
\hline & 0.05 & 13.76 & 12.37 & 17.37 \\
\hline & 0.5 & 0 & 0 & 0 \\
\hline \multirow{3}{*}{ Cetyltrimethyl ammonium bromide (CTAB)/cationic } & 0.005 & 12.78 & 14.34 & 19.25 \\
\hline & 0.05 & 8.48 & 7.89 & 9.51 \\
\hline & 0.5 & 0 & 0 & 0 \\
\hline \multirow{3}{*}{ Cocoanut fatty acid diethanolamide (CFAD)/nonionic } & 0.005 & 27.23 & 30.59 & 32.27 \\
\hline & 0.05 & 17.87 & 18.65 & 20.92 \\
\hline & 0.5 & 2.01 & 6.08 & 6.64 \\
\hline \multirow{3}{*}{ Dodecyl dimethyl betaine (DDB)/amphoteric } & 0.005 & 32.03 & 34.01 & 37.75 \\
\hline & 0.05 & 23.41 & 25.11 & 25.81 \\
\hline & 0.5 & 3.90 & 6.18 & 7.04 \\
\hline
\end{tabular}

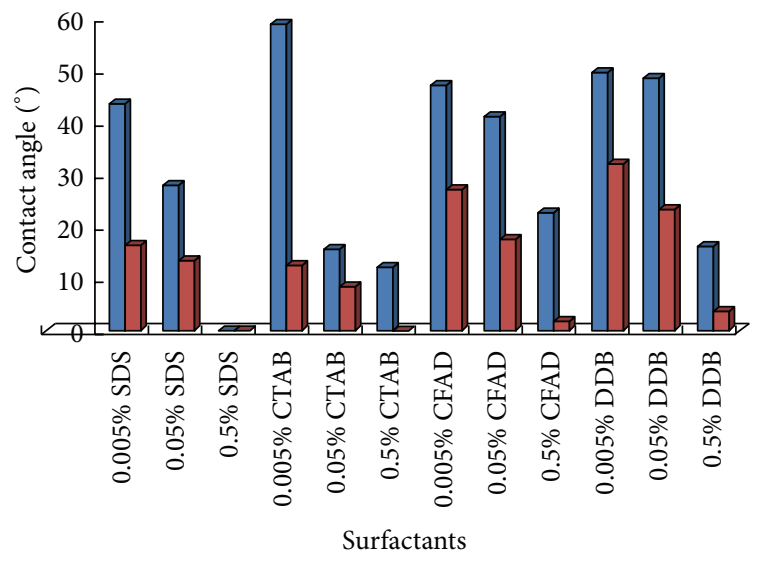

Coal pieces compressed with particle milled for $30 \mathrm{~s}$

Polished coal surface

FIGURE 6: Comparison chart of the contact angles between polished coal surface and coal piece surface. no matter what kind of surfactant solution is, with the increase in coal porosity and the decrease in volume percentage of the ultramicropore (aperture $<10 \mathrm{~nm}=100 \AA$ ), the contact angle tends to gradually decrease. The main reason for this phenomenon is that coal's increasing porosity and decreasing aperture dispersity help improve the surfactant's capacity to seepage into coal pores, thus increasing wetting effects to coal;

(2) anionic and cationic surfactants have better wetting ability to the natural coal surface than nonionic and amphoteric surfactants. When reagents (SDS and CTAB) formed contact angles on natural coal surface, their average values are $10.16^{\circ}$ and $7.09^{\circ}$, which are smaller than $15.70^{\circ}$ and $19.78^{\circ}$ formed by reagents (CFAD and DDB);

(3) obviously, the contact angle measured on the polished coal surface is smaller than that on the compressed 


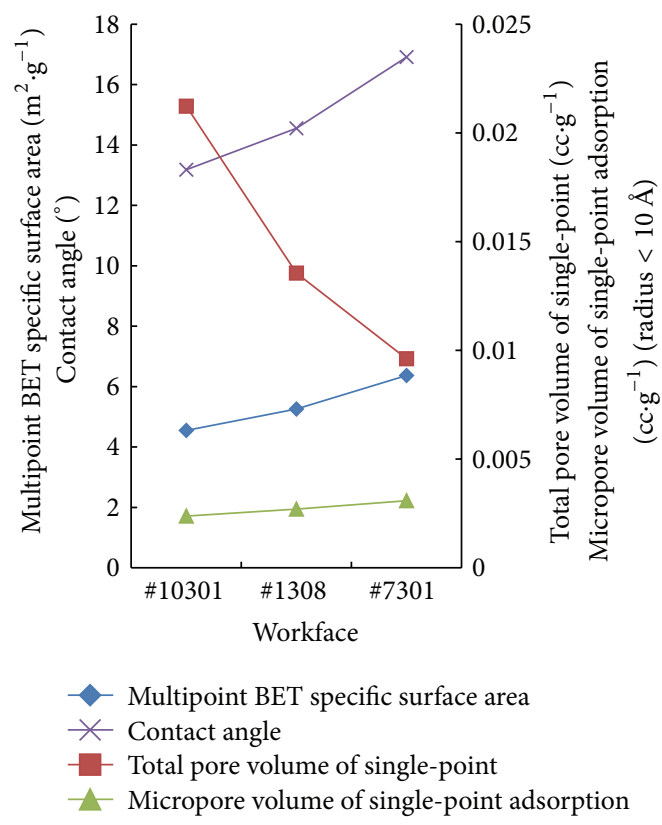

FIgURE 7: The relationships among contact angles and different porosity characteristics of different samples.

coal piece surface. For example, for coal samples from \#10301 working face, the average values of contact angles of four reagents (SDS, CTAB, CFAD, and DDB), which have three concentrations for each reagent, on polished coal surfaces are smaller than those on coal piece surfaces (coal particle milled for $30 \mathrm{~s})$ by $13.74^{\circ}, 21.91^{\circ}, 21.47^{\circ}$, and $18.51^{\circ}$ respectively.

\section{Conclusions}

In this paper, taking the gas coal in Xinglongzhuang coal mine as an example, the wetting characteristics of coal dust with different pore structure and particle size were investigated by contact angle experiments. The results showed that, with the gradual decreasing in the porosity of coal sample, the single-point total pore volume also decreases, while multipoint BET specific surface area increases. Single-point adsorption micropore volume increases with the decrease in single-point total pore volume. While the dispersion degree of ultramicropore decreases with the increase in porosity. With the increase in coal porosity and decrease in volume percentage of the ultramicropore (aperture $<10 \mathrm{~nm}=100 \AA$ ), the contact angle tends to decrease, and the coal is more easy to get wetted. In view of gas coal dust used in the experiment, only when the surface tension of the surfactant solution was less than $45 \mathrm{mN} / \mathrm{m}$ could the coal be fully wetted. And surfactants of anion and cation (SDS and CTAB) have better wetting ability than nonionic and amphoteric one (CFAD and DDB) regardless of compressed or polished coal pieces.

\section{Competing Interests}

The authors declare that they have no competing interests.

\section{Acknowledgments}

This work was financially supported by State Key Program of National Natural Science of China (Grant no. U1261205); National Natural Science Foundation of China (Grant no. 51474139); SDUST Research Fund (Grant no. 2014JQJH106); China Postdoctoral Science Foundation Funded Special Project (Grant no. 2016T90642); China Postdoctoral Science Foundation Funded Project (Grant no. 2015M570602); Open Fund of the Key Laboratory of Coal Mine Gas and Fire Prevention and Control of Ministry of Education (Grant no. 2015KJZX02); Science and Technology Project of Huangdao District, Qingdao (Grant no. 2014-1-30); Qingdao Postdoctoral Applied Research Project (Grant no. 2015194); Open Fund of the Key Laboratory of Safety and High-Efficiency Coal Mining of Ministry of Education (Grant no. JYBSYS2014105); The Key Technology Projects of Chinese State Administration of Work Safety for Preventing Major Safety Production Accidents (Grant no. Shandong-0083-2015AQ).

\section{References}

[1] H. Wang, D. Wang, W. Ren, X. Lu, F. Han, and Y. Zhang, "Application of foam to suppress rock dust in a large crosssection rock roadway driven with roadheader," Advanced Powder Technology, vol. 24, no. 1, pp. 257-262, 2013.

[2] H. Wang, D. Wang, Y. Tang, B. Qin, and H. Xin, "Experimental investigation of the performance of a novel foam generator for dust suppression in underground coal mines," Advanced Powder Technology, vol. 25, no. 3, pp. 1053-1059, 2014.

[3] G. Zhou, W.-M. Cheng, C.-C. Xu, and W. Nie, "Characteristic analysis of ${ }^{13} \mathrm{C}$-NMR for the wettability difference of coal dust with diverse degrees of metamorphism," Journal of the China Coal Society, vol. 40, no. 12, pp. 2849-2855, 2015.

[4] G. Zhou, J. Xue, W.-M. Cheng, W. Nie, and H. Wang, "Effect of stacking structure on the wettability of coal dust based on X-ray diffraction experiment," Chinese Journal of Engineering, vol. 37, no. 12, pp. 1535-1541, 2015.

[5] W. Nie, Research on the Airborne Dust Migration Rule and Inhibition Technology in Mechanized Excavation Face, Shandong University of Science and Technology, Qingdao, China, 2013.

[6] T. Murata, "Wettability of coal estimated from the contact angle," Fuel, vol. 60, no. 8, pp. 744-746, 1981.

[7] A. Gosiewska, J. Drelich, J. S. Laskowski, and M. Pawlik, "Mineral matter distribution on coal surface and its effect on coal wettability," Journal of Colloid and Interface Science, vol. 247, no. 1, pp. 107-116, 2002.

[8] A. Gosiewska, J. Drelich, M. Pawlik, J. Laskowski, and T. Eisele, "Contact angles on heterogeneous coal surface: the effect of mineral matter," Contact Angle, Wettability and Adhesion, vol. 2, pp. 177-199, 2002.

[9] D. P. Savitskyi, "Impact of the pH on angles of contact of water wettability of brown coal," Journal of Water Chemistry and Technology, vol. 37, no. 4, pp. 155-160, 2015.

[10] R. Ramesh and P. Somasundaran, "Chemical and wettability studies on coal, humic acid and cyclized humic acid," Fuel, vol. 68, no. 4, pp. 533-535, 1989.

[11] Q. Li, B. Lin, S. Zhao, and H. Dai, "Surface physical properties and its effects on the wetting behaviors of respirable coal mine dust," Powder Technology, vol. 233, pp. 137-145, 2013. 
[12] T. Chaturvedi, J. M. Schembre, and A. R. Kovscek, "Spontaneous imbibition and wettability characteristics of Powder River Basin coal," International Journal of Coal Geology, vol. 77, no. 1-2, pp. 34-42, 2009.

[13] A. Saghafi, H. Javanmard, and K. Pinetown, "Study of coal gas wettability for $\mathrm{CO}_{2}$ storage and $\mathrm{CH}_{4}$ recovery," Geofluids, vol. 14, no. 3, pp. 310-325, 2014.

[14] N. Siemons, H. Bruining, H. Castelijns, and K.-H. Wolf, "Pressure dependence of the contact angle in a $\mathrm{CO}_{2}-\mathrm{H}_{2} \mathrm{O}$-coal system," Journal of Colloid and Interface Science, vol. 297, no. 2, pp. 755-761, 2006.

[15] N. S. Kaveh, E. S. J. Rudolph, K.-H. A. A. Wolf, and S. N. Ashrafizadeh, "Wettability determination by contact angle measurements: hvbB coal-water system with injection of synthetic flue gas and $\mathrm{CO}_{2}$," Journal of Colloid and Interface Science, vol. 364, no. 1, pp. 237-247, 2011.

[16] R. Sakurovs and S. Lavrencic, "Contact angles in $\mathrm{CO}_{2}$-watercoal systems at elevated pressures," International Journal of Coal Geology, vol. 87, no. 1, pp. 26-32, 2011.

[17] N. Shojai Kaveh, K.-H. Wolf, S. N. Ashrafizadeh, and E. S. J. Rudolph, "Effect of coal petrology and pressure on wetting properties of wet coal for $\mathrm{CO}_{2}$ and flue gas storage," International Journal of Greenhouse Gas Control, vol. 11, pp. 91-101, 2012.

[18] F. O. Orumwense, "Estimation of the wettability of coal from contact angles using coagulants and flocculants," Fuel, vol. 77, no. 9-10, pp. 1107-1111, 1998.

[19] V. A. Arkhipov, D. Y. Paleev, Y. F. Patrakov, and A. S. Usanina, "Coal dust wettability estimation," Journal of Mining Science, vol. 50, no. 3, pp. 587-594, 2014.

[20] R. Ramesh and P. Somasundaran, "Centrifugal immersion technique for characterizing the wettability of coal particles," Journal of Colloid And Interface Science, vol. 139, no. 1, pp. 291294, 1990.

[21] W. G. Anderson, "Wettability literature survey-part 2: wettability measurement," Journal of Petroleum Technology, vol. 38, no. 11, pp. 1246-1262, 1986.

[22] F. F. O. Orumwense, "Wettability of coal-a comparative study," Scandinavian Journal of Metallurgy, vol. 30, no. 4, pp. 204-211, 2001.

[23] B. Holland, "Determination of both mesopores and macropores in three-dimensional ordered porous materials by nitrogen adsorption," Journal of Porous Materials, vol. 10, no. 1, pp. 1722, 2003.

[24] S. V. Potapov, A. A. Fomkin, V. A. Sinitsyn, A. V. Shkolin, and A. L. Pulin, "Nitrogen adsorption by microporous adsorbents in the range of high pressures and supercritical temperatures," Protection of Metals and Physical Chemistry of Surfaces, vol. 46, no. 5, pp. 519-523, 2010.

[25] G. Wang, K. Wang, and T. Ren, "Improved analytic methods for coal surface area and pore size distribution determination using $77 \mathrm{~K}$ nitrogen adsorption experiment," International Journal of Mining Science and Technology, vol. 24, no. 3, pp. 329-334, 2014.

[26] M. Nezafati, I. Sohn, J. B. Ferguson, J.-S. Park, K. Cho, and C.-S. Kim, "DFT study on the adsorption and absorption behaviors of liquid nitrogen in the $\mathrm{Mg}$ nano alloys synthesized from powder metallurgy," Computational Materials Science, vol. 105, pp. 1826, 2015.

[27] J. Pan, Q. Niu, K. Wang, X. Shi, and M. Li, "The closed pores of tectonically deformed coal studied by small-angle X-ray scattering and liquid nitrogen adsorption," Microporous and Mesoporous Materials, vol. 224, pp. 245-252, 2016.
[28] G. Zhou, Research of Theory about Dust Prevention by WaterCloud and Relevant Techniques for Fully-Mechanized Caving Coal Face, Shandong University of Science and Technology, Qingdao, China, 2009. 

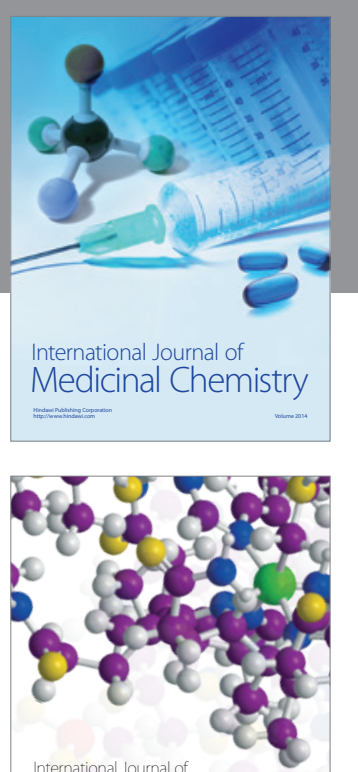

Carbohydrate Chemistry

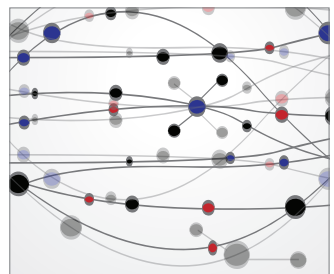

The Scientific World Journal
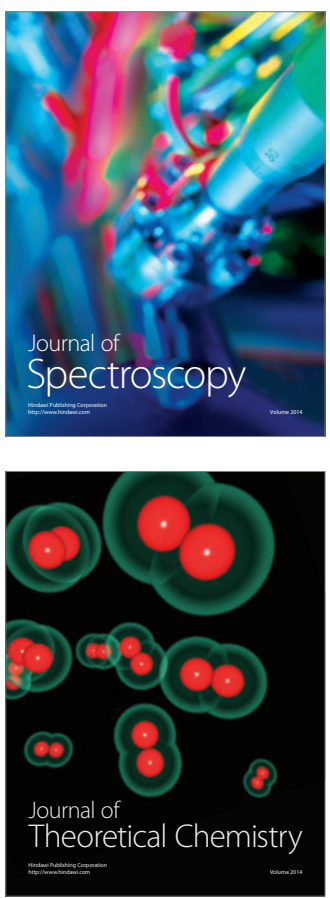
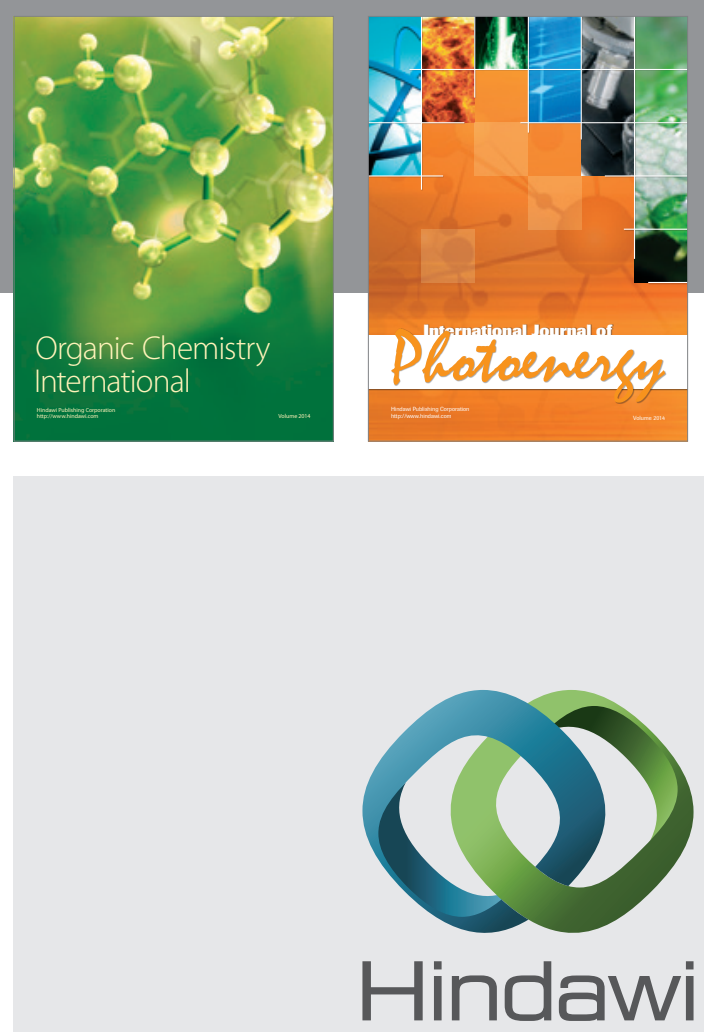

Submit your manuscripts at

http://www.hindawi.com

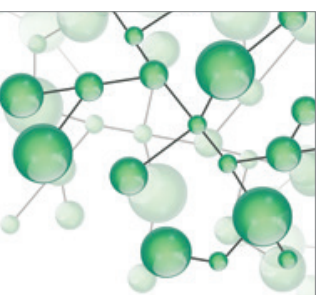

International Journal of

Inorganic Chemistry

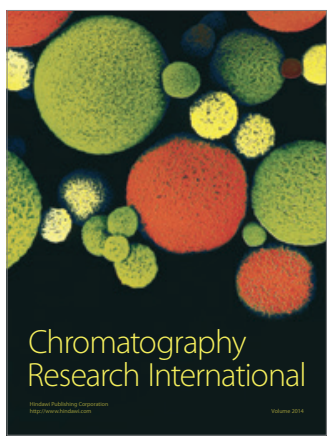

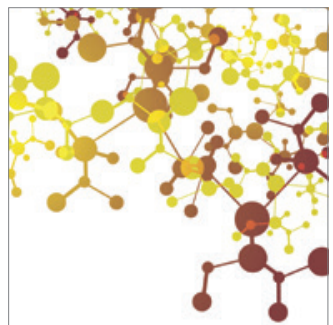

Applied Chemistry
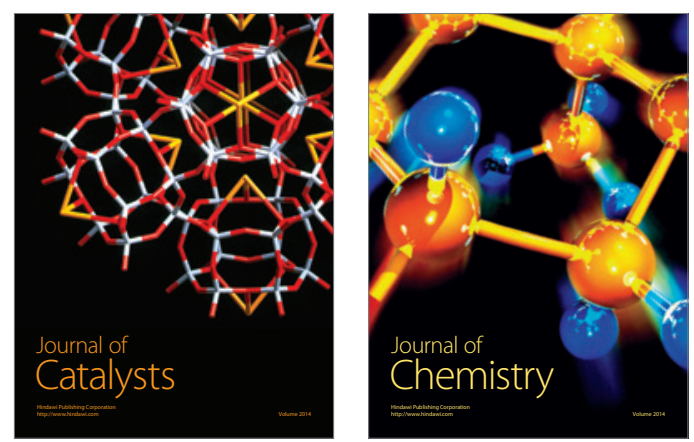
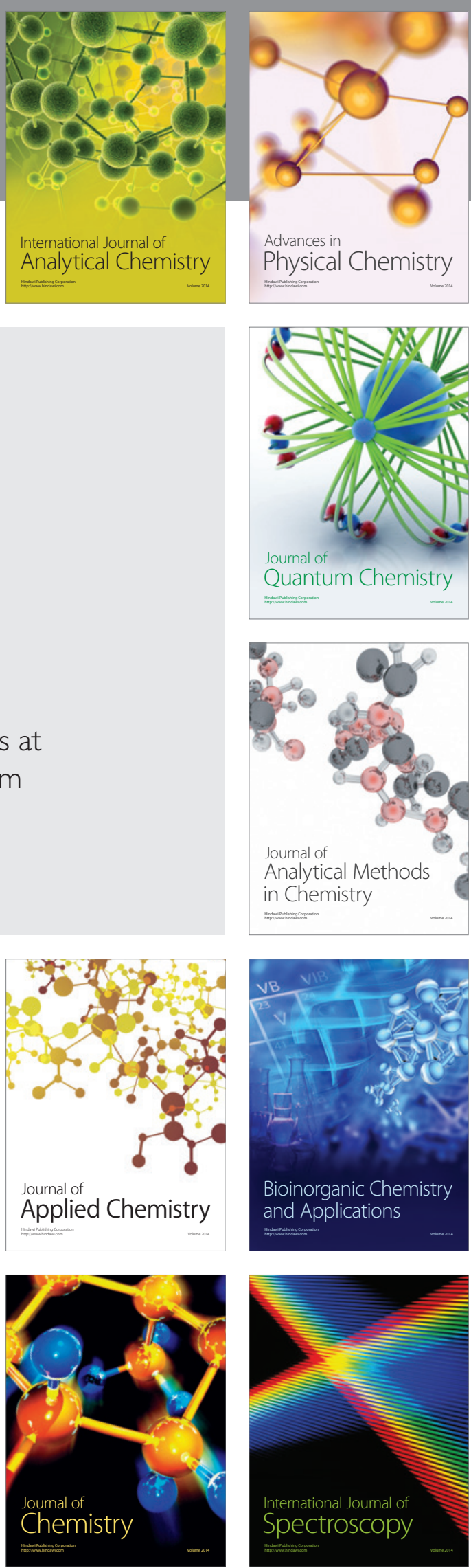